\title{
Efficacy of Ivermectin against Strongyloides Stercoralis in Humans
}

\author{
Koushin Shikiya, Nagisa Kinjo, Takeshi Uehara, Hiroyuki Uechi, Junichi Ohshiro, \\ Tamiki Arakaki, Fukunori Kinjo, Atsushi Saito, Mamoru IJu and Kazumine Kobari*
}

Okinawa Prefecture is an endemic area of Strongyloides stercoralis infection. Since treatment of this infection remains unsatisfactory, we evaluated the efficacy of ivermectin. Twentythree patients were treated with a single oral dose of ivermectin (mean $\pm \mathrm{SD}, 105.5 \pm 20.8 \mathrm{mcg}$ / $\mathrm{kg}$ of body weight), followed by a second dose two weeks later. The rate of cure was $85.7 \%$ at 2 weeks after the first treatment, and $90.5 \%$ at 2 weeks after the second treatment. Side effects occurred in 2 patients $(8.7 \%)$, but they were mild and transient. The results indicate that ivermectin might be useful and relatively safe for the therapy of Strongyloides stercoralis infection as an alternative to thiabendazole or mebendazole.

(Internal Medicine 31: 310-312, 1992)

Key words: Strongyloides stercoralis, ivermectin

\section{Introduction}

Although the incidence of Strongyloides stercoralis ( $S$. stercoralis) infection in Okinawa Prefecture was found to be less than $2 \%$ of the population when determined by traditional methods, it was proven to be $6.2 \%$ by a new technique, the agar plate method (1-3). Thiabendazole is strongly active in eradicating the organism, but it is well known that the rate of severe side effects is extremely high (4-6). Mebendazole is also active against $S$. stercoralis $(7,8)$, but a high incidence of liver disfunction was shown in our previous studies $(9,10)$. Ivermectin (IVM) has recently been found to be safe and effective for treatment of human onchocerciasis $(11-14)$ and lymphatic filariasis (15), but there are only two published reports concerning the efficacy of IVM against $S$. stercoralis in humans $(16,17)$. We therefore attempted to evaluate the efficacy of IVM against $S$. stercoralis infection.

\section{Materials and Methods}

\section{Study Subjects}

Twenty-three patients, 16 males and 7 females, with a positive $S$. stercoralis stool culture were included in this study. They ranged in age from 47 to 77 years with a mean age of 62 years.

\section{Treatment Protocol}

IVM (Merck Sharp \& Dohme Research Laboratories, New Jersey, USA) was given in a signle dose of $6 \mathrm{mg}$ one hour before breakfast and this treatment was repeated 2 weeks later. The dosages of IVM ranged from 68.4 to $160.0 \mathrm{mcg} / \mathrm{kg}$ of body weight.

Parasitological Examination

Fresh fecal samples, obtained before treatment, 2 weeks after the first treatment and 2 weeks after the second treatment, were examined using the agar plate method.

\section{Clinical Examination}

Clinical examination was done before treatment, 2 weeks after the initial treatment and 2 weeks after the second treatment. The examinations included a clinical history, a complete blood count, serum-chemistry studies, serological studies and urinalysis.

\section{Results}

\section{Pretreatment Evaluation}

Of the 23 patients, 9 patients $(39.1 \%)$ had no complaints before the treatment. Fourteen patients complained of some symptoms, including abdominal pain or borborygmus $(n=9$ patients), heartburn $(n=4)$, blurred vision $(n=3)$, arthralgia or lumbago $(n=3)$, headache $(\mathrm{n}=2)$, urinary frequency $(\mathrm{n}=2)$, diarrhea

From the First Department of Internal Medicine, Faculty of Medicine, University of the Ryukyus, Okinawa and * Ohama First Hospital, Okinawa

Received for publication Fcbruary 6, 1991; Accepted for publication August 7, 1991

Reprint requests should be addressed to Koushin Shikiya, M. D., the First Department of Internal Medicine, Faculty of Medicine, University of the Ryukyus, 207 Uehara, Nishihara Town, Okinawa 903-01, Japan 


\section{Efficacy of IVM against $S$. stercoralis}

$(\mathrm{n}=2)$, and constipation $(\mathrm{n}=2)$.

There were no abnormal laboratory data except eosinophilia and elevation of $\mathrm{IgE}$. The former occurred in $34.8 \%$ of the patients, and the latter was detected in $39.1 \%$ of the patients.

\section{Antiparasitic Effect}

The parasitological eradication rate at 2 weeks after the initial treatment was $85.7 \%$ (18 of 21 patients) and 2 weeks after the second course was $90.5 \%$ (19 of 21 patients). Three patients were uncured, their dosages of IVM were $133.3,78.9$ and $113.2 \mathrm{mcg} / \mathrm{kg}$ of body wt.

\section{Side Effects}

Twenty-one patients $(91.3 \%)$ experienced no side effects. One patient complained of borborygmus after the second treatment. Another patient complained of nausea, anorexia, dizziness or vertigo, blurred vision, and malaise after the first treatment, and itching after the second treatment. All symptoms were mild and required no treatment and subsided in a few days. None complained of severe dizziness or orthostatic hypotension. No abnormal laboratory findings were obtained in the hematological, serum chemistry or serological values after the treatment.

\section{Discussion}

Parasitological diagnosis is sometimes difficult because $S$. stercoralis larvae in the stool may be very few and inconsistently present. However, the new method, the agar plate method, is more efficient than the traditional methods (direct stool smear method, filter paper culture method and formalin-ether concentration method) (1-3).

$S$. stercoralis infection is usually asymptomatic and limited to the intestine. However, severe systemic disease due to $S$. stercoralis may develop in patients suffering from wasting diseases or malnutrition, or who are receiving immunosuppressive therapy or corticosteroids. Therefore, $S$. stercoralis infection should be treated with an effective and active drug. Although thiabendazole is effective against $S$. stercoralis, severe side effects occur (4-6).

We recently treated 24 patients with $100 \mathrm{mg}$ of mebendazole twice a day for 28 days in accordance with the reports of successful treatment of $S$. stercoralis, those reports also indicated only mild and infrequent side effects of the drug $(7,8)$. Although the eradication rate for our patients was $83.3 \%$, the incidence of liver disfunction was $71.4 \%$ of the patients (9). We then treated 47 patients for 5 day periods, repeating the treatment at 1,3 and 4 weeks following the initial 5 day treatment. The eradication rate was $100 \%$, but liver disfunction still occurred in $48.9 \%$ of the patients (10).

IVM is a semisynthetic macrocyclic lactone (a fermentation product of an actinomycete, Streptomyces avermitilis). Although it has been found to be microfilaricidal in clinical trials in humans, its precise action mechanism is unknown $(18,19)$. A series of clinical trials of IVM showed that a dose of $150 \mathrm{mcg} / \mathrm{kg}$ was safe and effective for onchocerciasis therapy (20).

There are only two reports concerning the efficacy of IVM against $S$. stercoralis in humans. Freedman and his colleagues (16) reported that IVM was $100 \%$ effective against strongyloidiasis ( 3 patients, with dosages of IVM ranging from 140 to $200 \mathrm{mcg} / \mathrm{kg}$ ). Naquira and colleagues (17) described results in which the cure rate at 30 days after treatment averaged $88 \%$ (101 patients, dosages of IVM were $50,100,150,200,100 \times 2,200 \times 2 \mathrm{mcg} / \mathrm{kg})$. Although the cure rates were significantly higher in recipients of doses $>150 \mathrm{mcg} / \mathrm{kg}$, there was no significant varience above that dose. In this study all patients were given $6 \mathrm{mg}$ of IVM and the dosage of uncured patients was $<150 \mathrm{mcg} / \mathrm{kg}$. Therefore, it would have been possible to eradicate the infection if those patients had received a higher dose of the drug.

We conclude that IVM is an effective drug for the treatment of $S$. stercoralis and that it also causes minimal side effects.

Acknowledgments: This study was supported by the Drug Research of Tropical Diseases Grant from the Ministry of Public Welfare, 1990, Japan and the 16th Oyama Health Foundation Grant, 1990, Japan.

\section{References}

1) Arakaki T, Hasegawa $H$, Asato $R$, et al. A new method to detect Strongyloides stercoralis from human stool. Japan J Trop Med Hyg 16: 11, 1988.

2) Arakaki T, Iwanaga M, Kinjo F, ct al. Efficacy of agarplate culture in detcction of Strongyloides stercoralis infection. J Parasitol 76: $425,1990$.

3) Asato R, Nakasone T, Arakaki T, Ikeshiro T. Prevalence of $S$. stercoralis infection among inhabitants of Okinawa by agar-plate method and evaluation of the mothods for detection. Ann Rep Okinawa Prefec Inst Publ Hlth 23: 66, 1989 (in Japanese).

4) Grove DI. Treatment of strongyloidiasis with thiabendazolc: an analysis of toxicity and effectiveness. Trans R Sco Trop Med Hyg 76: 114, 1982.

5) Pelletier LL. Chronic strongyloidiasis in World War 2 Far East cxprisoners of war. Am J Trop Med Hyg 33: 55, 1984.

6) Oyakawa $T$, Kuniyoshi $T$, Arakaki $T$, et al. New trial with thiabendazole for treatment of human strongyloidiasis. J J A Inf D 65: 304, 1991 (in Japanese).

7) Goldsmid JM. The use of mebendazole as a broad-spectrum anthelmintic in Rhodesia. S Afr Med 48: 2265, 1974.

8) Musgrave IA, Hawes RB, Jameson JL, Sloane RA, Quayle PA. Mcbendazole: evaluation of a new antihelminthic for trichuriasis, hookworm, and strongyloidiasis. Med J Aust 1: 404, 1979.

9) Shikiya $K$, Kuniyoshi $T$, Uechi $H$, et al. Treatment of strongyloidiasis with mebendazole and its combination with thiabendazole. J J A Inf D 64: 1408, 1990 (in Japanese).

10) Shikiya K, Kuniyoshi $T$, Uechi $H$, et al. Treatment of strongyloidiasis with mebendazole - long term eradication and new trials -. J J A Inf D 65: 433, 1991 (in Japanese).

11) Albiez EJ, Walter G, Kaiser A, et al. Histological examination of onchoccrcomata after therapy with ivermectin. Trop Med Parasit 39: 93, 1988. 


\section{Shikiya et al}

12) Greene BM, Taylor HR, Cupp EW, et al. Comparison of ivermectin and diethylcarbamazine in the treatment of onchocerciasis. N Engl J Med 313: 133, 1985.

13) Pacque M, Munoz B, Greene BM, et al. Safety of and compliance with community-based ivermectin therapy. Lancet 335: 1377, 1990.

14) Sole GD, Dadzie KY, Giese J, Remme J. Lack of adverse reactions in ivermectin treatment of onchocerciasis. Lancet 335: 1106, 1990.

15) Ottesen EA, Vijayasekaran V, Kumaraswami V, et al. A controlled trial of ivermectin and diethylcarbamazine in lymphatic filariasis. N Engl J Med 322: 1113, 1990.

16) Freedman DO, Zierdt WS, Lujan A, Nutman TB. The efficacy of ivermectin in the chemotherapy of gastrointestinal helminthiasis in humans. J Inf D 159: 1151, 1989.

17) Naquira $C$, Jimenez $G$, Guerra $J G$, et al. Ivermectin for human strongyloidiasis and other intestinal helminths. Am J Trop Med Hyg 40: 304, 1989.

18) Campbell WC, Fisher MH, Atapley EO, Albers-Schonberg G, Jacob TA. Ivermectin: a potent new antiparasitic agent. Science 221: 823, 1983.

19) Ette EI, Thomas WOA, Achumba JI. Ivermectin: a longacting microfilaricidal agent. DICP, Ann Pharmacother 24: 426, 1990.

20) Taylor HR, Greene BM. The status of ivermectin in the treatment of human onchocerciasis. Am J Trop Med Hy 41: 460, 1989. 